\title{
LA PROMOTION D’UNE IMAGE CORPORELLE POSITIVE CHEZ LES JEUNES
}

\author{
Isabelle Carrard, Sophie Bucher Della Torre, Michael Levine
}

S.F.S.P. | « Santé Publique »

2019/4 Vol. 31 | pages 507 à 515

ISSN 0995-3914

Article disponible en ligne à l'adresse :

https://www.cairn.info/revue-sante-publique-2019-4-page-507.htm

Distribution électronique Cairn.info pour S.F.S.P..

(C) S.F.S.P.. Tous droits réservés pour tous pays.

La reproduction ou représentation de cet article, notamment par photocopie, n'est autorisée que dans les limites des conditions générales d'utilisation du site ou, le cas échéant, des conditions générales de la licence souscrite par votre établissement. Toute autre reproduction ou représentation, en tout ou partie, sous quelque forme et de quelque manière que ce soit, est interdite sauf accord préalable et écrit de l'éditeur, en dehors des cas prévus par la législation en vigueur en France. Il est précisé que son stockage dans une base de données est également interdit. 


\title{
La promotion d'une image corporelle positive chez les jeunes
}

\section{Promoting a positive body image in young people}

\author{
Isabelle Carrard ${ }^{1}$, Sophie Bucher Della Torre ${ }^{1}$, Michael Levine ${ }^{2}$
}

\section{$[\rightarrow$ Résumé}

Introduction : Le concept d'image corporelle positive est récent. Il comprend plusieurs facettes, notamment l'acceptation de son corps et la capacité à en prendre soin avec des méthodes sans risque. Le but de cet article est d'expliquer le concept d'image corporelle positive, son importance, et d'effectuer une revue des interventions de prévention et de promotion de la santé potentiellement efficaces pour son développement.

Méthode : Nous avons effectué une revue narrative qui regroupe les dernières publications synthétisant l'utilité du concept et l'efficacité de programmes de prévention pouvant contribuer au développement d'une image corporelle positive chez les jeunes. Résultats : Le développement d'une image corporelle positive est envisagé comme facteur-clé qui permettrait une prévention intégrée de la totalité du spectre des problèmes de poids et de conduites alimentaires chez les jeunes, ainsi que d'autres problématiques telles que la dépression. Trois approches d'intervention de prévention développées pour cibler le facteur de risque qu'est l'insatisfaction corporelle pourraient s'avérer utiles pour promouvoir certains aspects d'une image corporelle positive. Il serait recommandé d'adopter une optique de prévention universelle et écologique, afin d'uniformiser les messages reçus dans l'ensemble de l'environnement social sur un comportement alimentaire sain, un style de vie actif et le respect de la diversité des silhouettes et de la beauté.

Conclusion : Cette revue met en évidence l'importance d'une image corporelle positive pour la santé publique, ainsi que les différentes interventions de prévention qui ont prouvé leur efficacité auprès des jeunes, afin que les futurs développements puissent s'appuyer sur ce qui a déjà été accompli.

Mots-clés: Image corporelle; Prévention; Troubles des conduites alimentaires ; Obésité ; Promotion de la santé.

\section{$\hookrightarrow$ Abstract}

Introduction: Positive body image is a recent concept that includes several facets, such as the acceptance of one's body and the ability to take care of it with safe, nurturing methods. The purpose of this article is to explain this concept and its importance, and to review prevention and health promotion interventions that might be effective for its development.

Method: We conducted a narrative review, including the latest publications that summarize the concept's usefulness and the effectiveness of prevention programs that may contribute to the development of positive body image in youth.

Results: The development of a positive body image is proposed as a key factor that would allow integrated prevention of the entire spectrum of weight and eating issues in young people, as well as other problems such as depression. Three approaches used for prevention interventions developed primarily to target body dissatisfaction as a risk factor might promote certain aspects of positive body image. It would be advisable to adopt a universal and ecological prevention approach, in order to standardize the messages received throughout the social environment regarding healthy eating, an active lifestyle, and respect for the diversity of weight, shape, and beauty.

Conclusion: This review highlights the importance of positive body image for public health, as well as the various preventive interventions that have proven effective for youth and young adults, so that future developments can build on what has already been performed.

Keywords: Body image; Prevention; Eating disorders; Obesity; Health promotion.

\footnotetext{
${ }^{1}$ Filière Nutrition et diététique, Haute École de santé, Haute École Spécialisée de Suisse occidentale HES-SO - Genève.

2 Department of Psychology, Kenyon College, Gambier, OH - USA - États-Unis. 


\section{Introduction}

\section{L'image corporelle positive}

Le concept d'«image corporelle positive » a émergé récemment comme étant un facteur associé au bien-être psychologique et favorisant les comportements de santé. L'image corporelle positive ne se résume pas à une absence d'insatisfaction corporelle, mais représente un construit plus complexe. Tylka et Wood-Barcalow [1] en ont détaillé les caractéristiques saillantes, qui sont présentées dans le tableau I. Les travaux sur ce concept ont notamment été influencés par le mouvement de la psychologie positive [2]. Celui-ci stipule que se focaliser sur l'éradication des fonctionnements négatifs ou des facteurs de risque, comme cela a été longtemps conçu en prévention et traitement des problèmes de santé mentale, ne créait au mieux qu'un état neutre. Cet état serait fragile et vulnérable à la rechute sous le poids des événements de vie contraires. Cultiver et promouvoir un fonctionnement positif chez les gens, en revanche, renforceraient leur résilience au-delà d'une simple atténuation ou absence de mécanismes négatifs, et protégeraient ainsi la santé psychique, même en cas de circonstances difficiles.

Tableau I : Quelques caractéristiques d'une image corporelle positive (tirées de Tylka et Wook-Barcalow) [1]

Accepter son corps, avoir une sensation de confort même sans être complètement satisfait-e de tous ses aspects, respecter le fait qu'il soit unique.

Conceptualiser la beauté de manière variée, prendre conscience qu'elle provient aussi de caractéristiques de la personnalité.

Focaliser sur les atouts de son corps plutôt que sur ses déficiences, sur ce qu'il nous permet d'effectuer, entretenir la confiance et l'affirmation de soi.

Investir de manière adaptée dans son apparence, prendre soin de soi, se mettre en valeur par des méthodes sans risque.

Développer un filtre protecteur de manière à se protéger : accepter les informations qui sont cohérentes avec une image corporelle positive et savoir reconnaître et rejeter les messages menaçants, provenant notamment des médias ou de l'entourage.

Avoir une flexibilité permettant de prendre soin de soi plutôt que d'avoir honte quand on est néanmoins atteint émotionnellement par des messages négatifs concernant son corps.

\section{Pourquoi s'intéresser à l'image corporelle en santé publique?}

La recherche a montré que l'insatisfaction corporelle augmentait significativement le risque de développer un trouble des conduites alimentaires, un comportement alimentaire malsain ou un style de vie sédentaire $[3,5]$. Une étude longitudinale de cinq ans, menée sur plus de 2500 adolescents (moyenne âge au temps $1=14,8$ ans, écart-type $=1,6$ ) a montré que, tant chez les filles que chez les garçons, l'insatisfaction corporelle prédisait des comportements de contrôle du poids malsains, des pertes de contrôle alimentaire ainsi qu'un niveau bas d'activité physique [6]. Ces résultats sont concordants avec des recherches antérieures revues par Stice [4], montrant que l'insatisfaction corporelle était un prédicteur robuste d'apparition et de maintien des troubles des conduites alimentaires chez les jeunes filles. Ainsi, comme souligné par Neumark-Sztainer et ses collègues, contrairement à la croyance populaire, le fait d'être insatisfait de son poids ou de sa silhouette ne réussit pas à motiver les gens à adopter un style de vie plus sain [6]. Les mesures mises en place en santé publique pour favoriser une alimentation saine et une activité physique auraient plus de chance d'atteindre leurs objectifs si elles étaient complétées par des actions éclairées et fondées sur des preuves concernant le développement d'une image corporelle positive.

De plus, l'insatisfaction corporelle est associée, voire prédictive dans certaines études, d'une estime de soi basse [7], de détresse émotionnelle [8], de dépression [7, 9] ou d'anxiété sociale [10]. L'insatisfaction corporelle a été également mise en lien avec des comportements à risque pour la santé, tels que les rapports sexuels non protégés [11] ou l'initiation du tabagisme [12], bien que les évidences soient mixtes concernant ce point [13]. Promouvoir une image corporelle positive à l'adolescence pourrait servir de facteur protecteur et ainsi prévenir ou atténuer de nombreux comportements malsains pouvant apparaitre durant cette étape de vie $[14,15]$. En effet, l'adolescence est une période cruciale à cause de l'intersection entre le développement physique, l'évolution de l'image corporelle et la formation de son identité. En tant que telle, il s'agit d'une période à risque, particulièrement pour les jeunes filles qui font face à une intensification des attentes sociales, à une objectification accrue de leur corps et, trop souvent, à une pression augmentée de la part des pairs, de la famille et des médias concernant le contrôle de sa faim, l'idéalisation de la minceur et le mépris de la graisse corporelle [16]. En parallèle, il s'agit également d'une période durant laquelle les garçons en développement sont influencés par 
des pressions socio-culturelles qui les orientent vers l'importance de la musculature, de la domination physique et de l'atteinte de la minceur (éviter à tout prix d'avoir de la graisse corporelle) [17]. Les études menées dans les différents pays francophones montrent que les jeunes issus de ces régions sont soumis aux mêmes pressions sur le corps que dans les pays anglo-saxons, en particulier concernant la minceur pour les jeunes filles et la musculature chez les jeunes garçons $[18,20]$.

\section{Pour une prévention intégrée}

L'amélioration de l'insatisfaction corporelle a été typiquement étudiée lors de l'évaluation des programmes de prévention des troubles des conduites alimentaires qui cherchent à faire décroître ce facteur de risque bien identifié [21]. En comparaison, sauf exceptions notables [p. ex. 22], les interventions de prévention du surpoids et de l'obésité chez les jeunes sont plutôt orientées sur la promotion d'un style de vie sain, comprenant une alimentation équilibrée et de l'activité physique. En général, l'effet possible de ces interventions sur l'image corporelle ou les comportements alimentaires problématiques n'est pas évalué [21]. Face aux développements parallèles dans ces deux champs qui collaborent peu malgré leurs points de convergence, des auteurs ont formulé des inquiétudes, par exemple qu'étiqueter le surpoids comme étant une menace à éviter risquait de promouvoir la " grossophobie » ainsi que la désirabilité sociale de la minceur que la société accentue déjà trop [23]. Au plan de la santé publique, certaines campagnes qui ciblent l'obésité pourraient laisser penser que la perte de poids et/ou l'évitement strict d'une prise de poids sont souhaitables pour tout un chacun et ainsi stigmatiser la graisse corporelle et les personnes en surpoids ; ce qui pourrait augmenter le risque d'insatisfaction corporelle au sein de la population générale [24]. Encourager les régimes restrictifs ou classer les aliments en « bons » et « mauvais » a été vu comme pouvant favoriser le développement ou le maintien des troubles des conduites alimentaires par les chercheurs et les thérapeutes de ce champ [25]. En fait, puisque les programmes de prévention de l'obésité chez les enfants n'incluent, en général, pas de mesures de santé psychologique ou de comportement alimentaire, Sanchez-Carracedo et al. [21] relèvent qu'il n'est actuellement pas possible de se positionner clairement sur des effets possiblement délétères.

$\mathrm{Au}$ vu des conséquences graves tant des troubles des conduites alimentaires que de l'obésité modérée à sévère, plusieurs chercheurs argumentent en faveur de messages et de programmes qui cibleraient de manière globale les problématiques en lien avec le poids et les conduites alimentaires chez les jeunes $[25,26]$; cela favoriserait la cohérence des messages de santé publique [27]. Intégrer ces approches demande de préciser quels sont les facteurs communs à l'ensemble de ces problématiques. Ceux-ci peuvent être regroupés en :

- Facteurs individuels psychologiques et comportementaux : préoccupations pour la silhouette et le poids, insatisfaction corporelle, comportements de contrôle du poids malsains, régimes, utilisation des médias, estime de soi.

- Facteurs interpersonnels et socio-environnementaux: environnement social (normes de minceur actuelles des cultures occidentales, discrimination du surpoids), environnement familial et interactions entre pairs (p. ex. remarques sur le poids émises dans la famille ou par les camarades), ou de l'école et de la communauté (p. ex. comportements des enseignants et des moniteurs de sport) [27].

On voit ainsi que, tant au niveau individuel qu'interpersonnel, l'insatisfaction corporelle, la pression sociale sur le poids et la silhouette, la non-acceptation de la graisse corporelle et de niveaux de poids plus élevés dans notre culture ainsi que les comportements de contrôle individuels et sociaux qui en découlent sont des facteurs communs qui pourraient avantageusement être ciblés par des programmes de prévention intégrée. Au-delà de réduire les facteurs de risque, établir des actions en faveur d'une image corporelle positive serait très probablement un moyen efficace de développement de facteurs protecteurs dans les champs de l'obésité, des troubles des conduites alimentaires et de la santé psychologique et physique en général $[14,15]$.

\section{Comment s'y prendre pour promouvoir une image corporelle positive?}

La majorité des programmes existants jusqu'à présent visent plutôt l'amélioration de l'image corporelle que la promotion d'une image corporelle positive. Ces interventions ont été le plus souvent développées dans le champ de la prévention des troubles du comportement alimentaire. La plupart d'entre elles avaient pour objectif de réduire le facteur de risque qu'est l'insatisfaction corporelle, plutôt que d'appliquer une approche "promotion de la santé», ce qui impliquerait une reconnaissance de l'importance et de la complexité des concepts de santé et d'image corporelle positive telle que décrits plus haut [21]. Néanmoins le 
recoupement, même partiel, des concepts d'insatisfaction corporelle et d'image corporelle positive justifie un intérêt pour ces recherches afin de construire, sur les connaissances déjà acquises, les approches les plus susceptibles d'être efficaces.

Plusieurs revues systématiques de la littérature, y compris des méta-analyses, se sont intéressées à l'effet des programmes de prévention des troubles des conduites alimentaires sur l'insatisfaction corporelle [p. ex. 5, 28, 29]. Ces revues ont mis en évidence que les approches interactives plutôt que didactiques, les interventions multisessions plutôt que session unique, et les interventions ciblant les jeunes filles adolescentes ou jeunes femmes plutôt qu'une audience mixte et plus jeune avaient le plus d'impact. Les tailles d'effet sont petites pour les programmes de prévention universelle, c'est-à-dire ceux conçus pour un échantillon relativement large d'adolescents à niveaux de risque mixtes sans critère d'exclusion; elles sont plus petites que les tailles d'effet modérées des programmes de prévention sélective, qui ciblent des personnes non symptomatiques mais considérées à haut risque, ou celles des programmes de prévention indiquée, ciblant des personnes à très haut risque de développer une pathologie à cause de la présence de précurseurs clairs ou de symptômes (voir Mrazek \& Haggerty [30] pour les détails sur le spectre d'intervention en santé mentale).

De nombreux auteurs soutiennent que la prévention universelle est particulièrement nécessaire dans le champ des troubles des conduites alimentaires [31, 32]. En effet, même si l'apparition des troubles des conduites alimentaires se produit généralement durant l'adolescence, la construction de l'image corporelle débute beaucoup plus tôt. Ceci suggère fortement qu'il est important de développer des mesures de prévention chez les enfants jeunes. Comme relevé par Levine et Smolak [31], cela ne fait pas de sens d'attendre l'apparition de facteurs de risque significatifs, sans parler de symptômes, avant de réaliser des actions de « prévention ». En outre, comme Austin [33] l’a démontré dans son application du Paradoxe de Rose, lorsqu'il s'agit de facteurs de risque plus ou moins normalement distribués au sein d'une population, un accent mis sur les individus à risque élevé ou très élevé conduit à « manquer » la majorité des personnes qui développent le trouble, car il y a relativement peu de personnes à haut risque. Comme on peut s'y attendre, et comme relevé précédemment, les programmes de prévention universelle testés avec des enfants plus jeunes et une population mixte ont produit des effets de taille moindre comparés aux programmes visant une réduction des facteurs de risque, voire des symptômes déjà présents, comme en prévention sélective et indiquée [31]. La prévention universelle reste cependant le moyen le plus efficace d'avoir un impact durable auprès d'une large tranche de la population et d'agir en amont du développement des facteurs de risque.

\section{Tour d'horizon d'une sélection d'interventions}

De nombreux programmes ont été conçus pour être prodigués dans le milieu scolaire qui permet d'atteindre les jeunes facilement. Les programmes développés et testés peuvent utiliser des approches très différentes, comme cela est par exemple mis en lumière dans le tableau II, qui représente une catégorisation proposée par Yager et al. [34] à partir de revues de littérature antérieures. Ces auteurs ont effectué la seule revue de littérature systématique qui s'est focalisée sur les interventions de prévention universelle ayant ciblé et évalué l'amélioration de l'image corporelle spécifiquement dans le contexte scolaire.

Pour cette revue systématique, Yager et al. [34] ont effectué leurs recherches dans les bases de données électroniques EBSCOhost, Medline, PsycINFO, Current Contents et Google Scholar, dans les numéros du journal Body Image parus entre 2008 et 2012 et les bibliographies des précédentes revues, systématiques ou non, et méta-analyses, identifiées au cours de leurs recherches. Des critères de sélection stricts ont été appliqués afin de faciliter la comparaison entre les programmes : l'audience consistait en pré-adolescents et adolescents âgés de 12 à 18 ans; les participants n'étaient pas présélectionnés et regroupés en fonction de facteurs de risque ; les études étaient publiées après 2000 dans un journal avec comité de lecture; les programmes étaient délivrés en face-à-face durant les heures de cours; l'évaluation du programme incluait une comparaison avec un groupe contrôle et au moins une mesure de satisfaction corporelle pré-post. Sur 42 études identifiées, les auteurs ont sélectionné 16 études évaluant 15 programmes, et exclu 26 études qui ne remplissaient pas leurs critères d'inclusion. Sur les 15 programmes recensés, sept programmes $(46,7 \%)$ ont conduit à une amélioration de l'image corporelle entre les mesures pré et postintervention, avec en moyenne une taille d'effet petite. Les deux seuls programmes qui se sont révélés efficaces après un suivi de trois mois [35], de six mois et de 30 mois [36] utilisaient, comme base pour leur intervention, l'éducation aux médias et l'acquisition de compétences pour résister à la pression socioculturelle des pairs concernant les idéaux de beauté et de minceur. Des approches qui pourraient sembler être au départ pertinentes (p. ex. la psychoéducation sur les troubles des conduites alimentaires) n'amènent 
Tableau II : Catégorisation des interventions utilisées dans les programmes de prévention ciblant l'image corporelle telle qu'utilisée par Yager et al. [34]

\begin{tabular}{|c|c|}
\hline Dénomination & Description brève \\
\hline Psychoéducation sur les TCA & Fournir de l'information sur les troubles des conduites alimentaires. \\
\hline Psychoéducation sur l'image corporelle & Fournir de l'information sur l'image corporelle. \\
\hline Facteurs développementaux & $\begin{array}{l}\text { Discussions sur les changements qui se produisent lors de la puberté, comme la prise } \\
\text { de poids. }\end{array}$ \\
\hline Amélioration des compétences d'estime de soi & Enseignement de stratégies pour améliorer son estime de soi. \\
\hline Compétences pour gérer son poids sainement & Enseignement de pratiques saines de contrôle du poids. \\
\hline Éducation aux médias & Développement de compétences d'analyse et de pensée critique sur le contenu des médias. \\
\hline Compétences pour faire face et gérer le stress & Enseignement de compétences de gestion du stress. \\
\hline Compétences d'acceptation du corps & Promotion de compétences d'acceptation de son corps. \\
\hline Dissonance cognitive & $\begin{array}{l}\text { Discussion et réflexion autour du coût qu'il y a à poursuivre les idéaux de minceur promus } \\
\text { par la société. }\end{array}$ \\
\hline Compétences socioculturelles entre pairs & Réduction des influences entre pairs (moqueries, comparaison, discours sur le poids...). \\
\hline
\end{tabular}

pas toujours de résultats quant à l'amélioration de l'image corporelle, ce qui montre l'importance de l'évaluation. Il ressort également de cette analyse qu'aucun des programmes sélectionnés pour cette revue ne s'est avéré efficace à la fois pour les garçons et les filles quand ils y participaient ensemble. Dans les settings mixtes, on observe une amélioration de l'image corporelle des garçons seulement [36, 37]. Les interventions évaluées dans la revue systématique de Yager et al. [34] étaient toutes anglophones. À titre informatif, nous pouvons citer comme exemple une intervention francophone Bien dans sa tête, bien dans sa peau qui a été développée et évaluée avec des effets positifs au Canada [38]. Cette intervention est composée d'ateliers qui, dans le cadre de l'étude, étaient dispensés par les infirmières scolaires, et qui visaient au développement de comportements en faveur de la santé et d'attitudes favorisant l'estime de soi et le respect de la diversité corporelle.

Nous avons choisi trois approches à présenter ici plus en détails : l'éducation aux médias, les interventions basées sur la dissonance cognitive et le programme pour un poids de santé. Nous les avons sélectionnées car il s'agit d'interventions basées sur une théorie claire et fondée, et parce que plusieurs études ont montré qu'elles avaient des effets désirables, à court et à long terme, sur des facteurs de risque tels que l'insatisfaction corporelle, l'internalisation des idéaux de minceur ou les préoccupations pour la silhouette et le poids $[22,36]$. Finalement, ces interventions permettent de s'occuper d'une partie des composantes d'une image corporelle positive décrites dans le tableau I, comme l'acceptation de son corps, une conceptualisation variée de la beauté et le rejet des messages délivrés par les médias valorisant la minceur et l'objectification du corps [15].

\section{Éducation aux médias}

L'éducation aux médias a pour but d'enseigner aux jeunes à décoder les médias, par exemple les messages publicitaires, afin de renforcer leur esprit critique sur les techniques de manipulation utilisées par les industries qui font les médias de masse, et sur les messages malsains qui sont transmis, par exemple en ce qui concerne le poids, les idéaux de beauté filiforme pour les jeunes filles et les femmes, les stéréotypes de genre et l'objectification du corps féminin. Cette approche a déjà été utilisée dans plusieurs champs de promotion de la santé [39]. Dans le cadre de la prévention des troubles des conduites alimentaires, les programmes axés sur l'éducation aux médias visent en général cinq objectifs [3 (chapitre 13), 39] :

1) Sensibiliser aux messages médiatiques concernant le poids, la silhouette idéale, le contrôle, le genre, ainsi qu'aux messages encourageant la gratification alimentaire immédiate, à manger de grandes quantités, etc.

2) Discuter et analyser l'impact de ces messages sur l'image corporelle, l'estime de soi, les relations avec les pairs, etc.

3) Présenter les trucs et astuces utilisés dans les médias pour créer des images idéales.

4) Générer des stratégies pour se protéger de tels messages.

5) Adopter une perspective activiste pour contester et modifier les messages médiatiques, par exemple en 
écrivant à l'industrie pour exprimer une opinion en tant que consommateur.

Les programmes d'analyse critique des médias encouragent les participants à être actifs en développant une variété de compétences d'analyse et de communication afin d'appliquer les concepts qu'ils apprennent. Wilksch et Wade [40] relèvent que l'éducation aux médias a également des bénéfices secondaires, tels que le fait de faire participer et d'engager activement les adolescents, garçons et filles, à améliorer les relations entre eux, en réduisant les moqueries et les commentaires liés au poids et en leur fournissant un ensemble de compétences pour améliorer leur estime d'eux-mêmes et leur sentiment d'autonomisation [40].

L'éducation aux médias est donc une approche qui convient à la prévention universelle et plusieurs programmes ont été évalués. Cependant, Paxton [41] relève que tous les programmes mis à disposition sur Internet ne sont pas efficaces. Elle cite une étude menée par Diedrichs et al. [42] qui n'ont pas trouvé de différence d'amélioration de la satisfaction corporelle entre des participants à une intervention de type éducation aux médias, promue par le Gouvernement britannique, et un groupe contrôle. L'intervention était possiblement trop courte, mais il a également été relevé qu'une partie des jeunes élèves se sentaient mal à l'aise avec des images utilisées qui étaient trop révélatrices. Cela met en évidence l'importance de prétester consciencieusement le matériel utilisé, même pour une approche par ailleurs validée.

\section{Les interventions basées sur la dissonance cognitive}

L'intervention dénommée "dissonance cognitive » a été développée par Stice et ses collègues et évaluée, initialement, dans plusieurs études de prévention sélective et/ou indiquée qui incluaient principalement des adolescentes et des jeunes femmes à risque [22]. La dissonance cognitive est un phénomène psychologique qui a été largement étudié pendant plus de 50 ans [43]. Il s'agit d'une tension psychique inconfortable qui survient quand des croyances importantes ne sont pas en accord les unes avec les autres ou avec les actions de la personne. Un aspect de la théorie est la prédiction que, si des circonstances externes ne sont pas clairement la cause d'un comportement dissonant (par exemple la recherche d'une récompense ou l'évitement d'une punition), pour réduire la dissonance, l'individu va modifier ses croyances pour qu'elles s'alignent avec son comportement [44]. Dans le cadre de la prévention des troubles des conduites alimentaires, les participantes sont encouragées à volontairement et publiquement, c'est-à-dire en face d'autres personnes, parler et agir à l'encontre des standards de beauté féminins internalisés qui promeuvent la minceur. Théoriquement, un tel comportement serait incohérent avec leurs propres croyances - largement répandues par ailleurs - en faveur de ces idéaux de minceur, ce qui crée de la dissonance et conduit les participantes à réduire leur internalisation de ces idéaux [44].

Le programme original, dénommé The Body Project [45], est décrit sur le site http://www.bodyprojectcollaborative. com/ qui présente le concept et en propose différentes versions. L'intervention est modulable mais généralement délivrée en 3-4 sessions d'une heure. The Body Project a également l'avantage de pouvoir être disséminé par des pairs qui ont suivi une formation, dans certains cas, donnée par d'autres pairs. Plusieurs études ont démontré que The Body Project pouvait être mis en œuvre de manière efficace pour des jeunes femmes universitaires avec une facilitation par les pairs [voir p. ex. 46]. À noter qu'une étude, qui a récemment évalué l'effet de The Body Project sur une audience à risque mixte en prévention universelle, a trouvé que la satisfaction corporelle des garçons s'améliorait, en comparaison d'un groupe contrôle [47]. Les résultats de cette étude amènent néanmoins de nouvelles questions; en effet, les filles ne profitaient pas de l'intervention comme elles en avaient régulièrement profité dans les études précédentes.

\section{Programme pour un poids de santé (Healthy Weight)}

Initialement, le programme Healthy Weight a été développé par Stice et ses collègues pour être utilisé comme intervention placebo contrôle et évaluer ainsi, de manière rigoureuse, The Body Project [48]. Dans le programme Healthy Weight, les participantes acquièrent des connaissances sur les déterminants du poids et sont guidées pour mettre en œuvre des modifications dans leur style de vie, comprenant une amélioration de leur alimentation et de leur niveau d'activité physique. Stice et ses collègues ont créé ce programme afin qu'il soit crédible, mais ils ne pensaient pas qu'il aurait un effet sur les facteurs de risque visés; en effet, les programmes psycho-éducatifs promouvant un style de vie sain n'avaient jamais produit d'effets constants et appréciables [5, 28]. Cependant, dès sa première évaluation, le programme Healthy Weight a démontré des effets bénéfiques similaires à ceux observés pour The Body Project, notamment en ce qui concerne la diminution de deux facteurs de risque importants pour les troubles des conduites alimentaires : l'internalisation des idéaux de minceur et l'insatisfaction corporelle [22]. Tout comme le programme The Body Project, Healthy Weight est un programme initialement évalué en prévention 
sélective-indiquée, sur une population de jeunes femmes à risque, avec des préoccupations relatives à leur poids, et peut également être délivré par des pairs de manière efficace [49].

\section{Prendre en compte les facteurs environnementaux}

Ces interventions de prévention, issues du champ des troubles des conduites alimentaires, ciblent les facteurs de risque individuel. Cependant, comme vu plus haut, les éléments conduisant au développement de facteurs de risque, tels qu'une image corporelle négative, opèrent à de multiples niveaux environnementaux, y compris au niveau de la famille, des pairs, des activités sportives ou de loisirs, de la communauté et en termes de valeurs et de politiques sociales [3]. Les influences entre pairs sont un niveau particulièrement important, qui inclut les comparaisons sociales et l'exposition directe au discours sur le poids ("fat talk» en anglais). Des programmes dits "écologiques», qui adoptent une visée plus large et ciblent ces différents niveaux environnementaux, permettraient une meilleure cohérence des messages reçus et le maintien, à plus long terme, des effets des interventions de prévention.

En exemple d'une intervention de prévention qui a ciblé le contexte ainsi que les individus, on peut citer New Moves $[50,51]$ (tout le matériel est accessible sur Internet à http://www.newmovesonline.com), un programme du champ de l'obésité qui a été conçu comme un moyen de prévention intégrée pour un large spectre de comportements alimentaires et de pratiques de contrôle du poids inadéquats. Ainsi, la première composante du programme était une classe d'activité physique pour jeunes filles sédentaires incluant des activités sur l'amélioration du comportement alimentaire et de l'image corporelle sans inciter à suivre des régimes. Le développement de New Moves a été conceptualisé à partir d'une analyse des besoins de ces jeunes filles. En conséquence, son développement ainsi que sa mise en œuvre a inclus les enseignants, ainsi qu'un petit module pour les parents. Le résultat est une approche cohérente améliorant l'environnement des jeunes filles. New Moves leur permet d'effectuer de l'activité physique dans un contexte où elles se sentent confortables et en sécurité, à l'abri des moqueries. Toute une nouvelle gamme d'activités et de possibilités leur est offerte par une variété d'intervenants adultes qui font office de modèles variés en termes de silhouette, de taille et d'ethnie. Dans une étude randomisée, New Moves a été testé dans six écoles et comparé à six écoles contrôle. En moyenne, le programme n'a pas produit de changement au niveau de l'indice de masse corporelle des participantes. Cependant, comme prévu, New Moves a amené des améliorations, maintenues à neuf mois, de l'activité physique, des habitudes alimentaires et de l'image corporelle, tout en réduisant les comportements problématiques de gestion du poids [51]. On voit ainsi que l'école est un milieu pertinent pour introduire cette thématique auprès des jeunes, qu'il s'agisse d'implémenter des interventions autonomes dispensées en classe, d'insérer les thématiques de la diversité corporelle et des comportements de santé au sein des différentes matières enseignées, ou de construire un environnement plus favorable au développement d'une image corporelle positive chez les jeunes.

En conclusion, pour permettre la cohérence, à différents niveaux écologiques, entre les messages et les programmes destinés aux jeunes, une coordination des différentes actions et du développement et de l'application de réglementations et de politiques publiques serait nécessaire pour assurer que les messages délivrés au public soient conçus dans le respect de l'image corporelle positive et préviennent les problématiques autour de l'image corporelle [41]. Si l'on se réfère au tableau I, par exemple, on pourrait envisager une politique qui dicterait que la documentation émise par les différents organismes, concernant l'alimentation et l'activité physique, doive être contrôlée afin d'assurer que ce matériel promeuve l'acceptation du poids et de silhouettes diverses, tout en encourageant un style de vie sain. Dans le même ordre d'idées, des entités, avec ou sans but lucratif, pourraient recevoir le mandat d'élaborer des recommandations qui encourageraient la promotion d'une image corporelle positive et la justice sociale, tout en vendant des produits et/ou en fournissant des services sociaux, en appliquant à la fois créativité artistique et principes de publicité performants. De même, les sites Web créés par les différentes institutions, les affiches ou tout matériel pourraient inclure des images et des symboles montrant des hommes et des femmes, des garçons et des filles, de poids et de silhouettes variés.

\section{Conclusion}

Cet article a pour but de mettre en évidence la nécessité d'intégrer la promotion d'une image corporelle positive dans les programmes de santé publique et d'explorer des approches possibles, basées sur celles qui ont déjà été développées et évaluées auprès des jeunes. En particulier, inclure l'image corporelle positive dans la prévention 
universelle a l'avantage de prévenir un large spectre de comportements délétères pour la santé : les désordres alimentaires, l'obésité, la dépression, les rapports sexuels non protégés ou non désirés ou la consommation de tabac, par exemple. Promouvoir une image corporelle positive chez des jeunes correspond à adopter une vision large de la promotion de la santé et représente, en particulier, une option efficace pour intégrer la prévention des troubles des conduites alimentaires et des problèmes autour du poids.

Il ressort des revues de littérature récentes que de nombreux programmes efficaces, ou potentiellement efficaces, ont déjà été développés, principalement en langue anglaise. Cela nous inciterait plutôt à ne pas consacrer de ressources à en développer de nouveaux, mais à utiliser ceux qui existent tout en adaptant le matériel selon la culture, l'âge de l'audience, le mode de transmission et le niveau de prévention désiré. En outre, au niveau de la recherche, proposer des réplications dans d'autres sites est extrêmement important pour évaluer la validité écologique d'un programme, or cela a rarement été fait. Les développements souhaitables devraient permettre de s'adresser à tous les âges et à des audiences mixtes : on manque, en effet, de programmes de prévention universelle efficaces pour les garçons et les filles ensemble. À cet égard, il reste très important de mieux comprendre quels sont les facteurs modérateurs en jeu pour pouvoir atteindre garçons et filles avec les mêmes interventions.

\section{Aucun conflit d'intérêts déclaré}

\section{Remerciements}

Ce travail a été réalisé grâce au soutien financier de Promotion Santé Suisse, du Département de la sécurité, de l'emploi et de la santé du Canton de Genève et de la Haute École de santé de Genève (HES-SO Genève).

\section{Références}

1. Tylka TL, Wood-Barcalow NL. What is and what is not positive body image? Conceptual foundations and construct definition. Body Image. 2015;14:118-29.

2. Seligman ME, Csikszentmihalyi M. Positive psychology. An introduction. Am Psychol. 2000;55(1):5-14.

3. Levine $M$, Smolak $L$. The prevention of eating problems and eating disorders. Theory, research, and practice. Mahwah, NJ: Lawrence Erlbaum Associates; 2006. 480 p.

4. Stice E. Risk and maintenance factors for eating pathology: a meta-analytic review. Psychol Bull. 2002;128(5):825-48.
5. Stice E, Shaw H, Marti CN. A meta-analytic review of eating disorder prevention programs: encouraging findings. Annu Rev Clin Psychol. 2007:3:207-31.

6. Neumark-Sztainer D, Paxton SJ, Hannan PJ, Haines J, Story M. Does body satisfaction matter? Five-year longitudinal associations between body satisfaction and health behaviors in adolescent females and males. J Adolesc Health. 2006;39(2):244-51.

7. Paxton SJ, Neumark-Sztainer D, Hannan PJ, Eisenberg ME. Body dissatisfaction prospectively predicts depressive mood and low self-esteem in adolescent girls and boys. J Clin Child Adolesc Psychol. 2006;35(4):539-49.

8. Johnson F, Wardle J. Dietary restraint, body dissatisfaction, and psychological distress: A prospective analysis. J Abnorm Psychol. 2005;114(1):119-25.

9. Stice E, Hayward C, Cameron RP, Killen JD, Taylor CB. Body-image and eating disturbances predict onset of depression among female adolescents: a longitudinal study. J Abnorm Psychol. 2000;109(3): 438-44.

10. Tantleff-Dunn S, Lindner DM. Body image and social functioning. In: Cash TF, Smolak L, editors. Body Image A Handbook of Science, Practice, and Prevention. 2nd ed. New York: The Guilford Press; 2011. pp. 263-70.

11. Schooler D. Early Adolescent Body Image Predicts Subsequent Condom Use Behavior Among Girls. Sex Res Soc Policy. 2013;10(1): 52-61.

12. Howe $\sqcup$, Trela-Larsen L, Taylor M, Heron J, Munafo MR, Taylor AE. Body mass index, body dissatisfaction and adolescent smoking initiation. Drug Alcohol Depend. 2017;178:143-9.

13. Kaufman AR, Augustson EM. Predictors of regular cigarette smoking among adolescent females: does body image matter? Nicotine Tob Res. 2008;10(8):1301-9.

14. Levine M, Smolak L. The role of protective factors in the prevention of negative body image and disordered eating. Eat Disord. 2016:24(1):39-46.

15. Piran N. New possibilities in the prevention of eating disorders: The introduction of positive body image measures. Body Image. 2015;14:146-57.

16. Levine M, Smolak L, Moodey AF, Shuman MD, Hessen LD. Normative developmental challenges and dieting and eating disturbances in middle school girls. Int J Eat Disord. 1994;15(1):11-20.

17. Limbers CA, Cohen LA, Gray BA. Eating disorders in adolescent and young adult males: prevalence, diagnosis, and treatment strategies. Adolesc Health Med Ther. 2018;9:111-6.

18. Dany L, Morin M. Image corporelle et estime de soi : étude auprès de lycéens français. B Psychol. 2010;63(5):321-34.

19. Image corporelle positive chez les jeunes en Suisse : une comparaison entre la Suisse alémanique $(2015)$ et la suisse romande (2016). Berne : Promotion Santé Suisse ; 2017. 11 p. (Feuille d'information; 25). [Visité le 28/06/2019]. En ligne: https:// promotionsante.ch/assets/public/documents/fr/5-grundlagen/ publikationen/ernaehrung-bewegung/faktenblaetter/Feuille_d_ information_025_PSCH_2017-10_-_L_image_corporelle_chez_ les_adolescents_en_Suisse.pdf.

20. Camirand $H$, Cazale L, Bordeleau M. Les élèves du secondaire sont-ils satisfaits de leur apparence corporelle ? Zoom santé, Institut de la statistique du Québec. 2015;49:1-8.

21. Sanchez-Carracedo D, Neumark-Sztainer D, Lopez-Guimera G. Integrated prevention of obesity and eating disorders: barriers, 
developments and opportunities. Public Health Nutr. 2012;15(12): 2295-309.

22. Stice E, Marti CN, Spoor S, Presnell K, Shaw H. Dissonance and healthy weight eating disorder prevention programs: long-term effects from a randomized efficacy trial. J Consult Clin Psychol. 2008;76(2):329-40.

23. Puhl R, Heuer CA. The stigma of obesity: a review and update. Obesity (Silver Spring). 2009;17(5):941-64.

24. Puhl R, Brownell KD. Bias, discrimination, and obesity. Obes Res. 2001;9(12):788-805.

25. Neumark-Sztainer D. Can we simultaneously work toward the prevention of obesity and eating disorders in children and adolescents? Int J Eat Disord. 2005;38(3):220-7.

26. Wilksch SM, Paxton SJ, Byrne SM, Austin SB, McLean SA, Thompson $\mathrm{KM}$, et al. Prevention Across the Spectrum: a randomized controlled trial of three programs to reduce risk factors for both eating disorders and obesity. Psychol Med. 2015;45(9):1811-23.

27. Neumark-Sztainer D, Wall MM, Haines JI, Story MT, Sherwood NE, van den Berg PA. Shared risk and protective factors for overweight and disordered eating in adolescents. Am J Prev Med. 2007;33(5): 359-69.

28. Stice $E$, Shaw $H$. Eating disorder prevention programs: a metaanalytic review. Psychol Bull. 2004;130(2):206-27.

29. Cororve Fingeret M, Warren CS, Cepeda-Benito A, Gleaves DH. Eating disorder prevention research: a meta-analysis. Eat Disord. 2006;14(3):191-213.

30. Mrazek PJ, Haggerty RJ. Reducing risks for mental disorders: Frontiers for preventive intervention research. Washington, DC: National Academy Press; 1994. 636 p.

31. Levine M, Smolak L. "What exactly are we waiting for?" The case for universal-selective eating disorders prevention programs. Int J Child Adolesc Health. 2008;1(4):295-304.

32. Bell MJ, Rodgers RF, Paxton SJ. Towards successful evidence-based universal eating disorders prevention: The importance of zooming out. Eat Behav. 2017;25:89-92.

33. Austin SB. Population-based prevention of eating disorders: an application of the Rose prevention model. Prev Med. 2001;32(3): 268-83.

34. Yager Z, Diedrichs PC, Ricciardelli LA, Halliwell E. What works in secondary schools? A systematic review of classroom-based body image programs. Body Image. 2013;10(3):271-81.

35. Richardson SM, Paxton SJ. An evaluation of a body image intervention based on risk factors for body dissatisfaction: a controlled study with adolescent girls. Int J Eat Disord. 2010;43(2):112-22.

36. Wilksch SM, Wade TD. Reduction of shape and weight concern in young adolescents: a 30-month controlled evaluation of a media literacy program. J Am Acad Child Adolesc Psychiatry. 2009;48(6): 652-61.

37. Richardson SM, Paxton SJ, Thomson JS. Is BodyThink an efficacious body image and self-esteem program? A controlled evaluation with adolescents. Body Image. 2009;6(2):75-82.

38. Blackburn M-Ë, Auclair J, Jean M, Dion J, Gagnon J, Brault M-C, et al. Évaluation des effets du programme d'intervention en milieu scolaire "Bien dans sa tête, bien dans sa peau ". Jonquière : ÉCOBESRecherche et transfert, Cégep de Jonquière; 2019.

39. Yager Z, O'Dea JA. School-based prevention. In: Smolak L, Levine MP, editors. The Wiley Handbook of Eating Disorders. vol. 2. Assessment, Prevention, Treatement, Policy, and Future Directions. Chichester, UK: John Wiley \& Sons, Ltd.; 2015. pp. 569-81.

40. Wilksch SM, Wade TD. Media literacy in the prevention of eating disorders. In: Smolak L, Levine MP, editors. The Wiley Handbook of Eating Disorders. vol. 2. Assessment, Prevention, Treatement, Policy, and Future Directions. Chichester, UK: John Wiley \& Sons, Ltd; 2015. pp. 610-24.

41. Paxton SJ. Social policy and prevention. In: Smolak L, Levine MP, editors. The Wiley handbook of eating disorders. vol. 2. Assessment, Prevention, Treatement, Policy, and Future Directions. Chichester, UK: John Wiley \& Sons, Ltd; 2015. pp. 655-68.

42. Diedrichs PC, Yager Z, Parskeva N, Halliwell E. Media Smart? Evaluating a government endorsed body image intervention for primary schools. Appearance Matters 5; Bristol, UK2012.

43. Festinger L. A theory of cognitive dissonance. Stanford, CA: Stanford University Press; 1957.

44. Becker CB, MacKenzie K, Stewart T. Cognitive and behavioral approaches to the prevention of eating disorders. In: Smolak $\mathrm{L}$, Levine MP, editors. The Wiley Handbook of Eating Disorders. vol. 2 Assessment, Prevention, Treatment, Policy, and Future Directions. Chichester, UK: John Wiley \& Sons, Ltd; 2015. pp. 597-609.

45. Stice $E$, Rhode $P$, Shaw $H$. The body project: A dissonance-based eating disorder prevention intervention. Facilitators guide. Updated ed. ed. New York: Oxford University Press; 2013. 148 p.

46. Kilpela LS, Hill K, Kelly MC, Elmquist J, Ottoson P, Keith D, et al. Reducing eating disorder risk factors: a controlled investigation of a blended task-shifting/train-the-trainer approach to dissemination and implementation. Behav Res Ther. 2014;63:70-82.

47. Kilpela LS, Blomquist K, Verzijl C, Wilfred S, Beyl R, Becker CB. The body project 4 all: A pilot randomized controlled trial of a mixedgender dissonance-based body image program. Int J Eat Disord. 2016;49(6):591-602.

48. Stice E, Chase A, Stormer S, Appel A. A randomized trial of a dissonance-based eating disorder prevention program. Int J Eat Disord. 2001;29(3):247-62.

49. Becker CB, Wilson C, Williams A, Kelly M, McDaniel L, Elmquist J. Peer-facilitated cognitive dissonance versus healthy weight eating disorders prevention: A randomized comparison. Body Image. 2010;7(4):280-8.

50. Neumark-Sztainer D, Flattum CF, Story M, Feldman S, Petrich CA. Dietary approaches to healthy weight management for adolescents: the New Moves model. Adolesc Med State Art Rev. 2008;19(3): 421-30, viii.

51. Neumark-Sztainer D, Friend SE, Flattum CF, Hannan PJ, Story MT, Bauer KW, et al. New moves-preventing weight-related problems in adolescent girls a group-randomized study. Am J Prev Med. 2010;39(5):421-32. 